\title{
On the Travelling Wave Solution for the Current-Driven Steady Domain Wall Motion in Magnetic Nanostrips under the Influence of Rashba Field
}

\author{
Vito Puliafito $^{1}$ and Giancarlo Consolo ${ }^{2}$ \\ ${ }^{1}$ Department of Matter Physics and Electronic Engineering, University of Messina, V.le F. Stagno D'Alcontres 31, \\ 98166 Messina, Italy \\ ${ }^{2}$ Department of Science for Engineering and Architecture, University of Messina, Contrada Di Dio, 98166 Messina, Italy
}

Correspondence should be addressed to Vito Puliafito, vpuliafito@unime.it

Received 31 March 2012; Revised 17 May 2012; Accepted 19 May 2012

Academic Editor: Roberto Zivieri

Copyright ( 2012 V. Puliafito and G. Consolo. This is an open access article distributed under the Creative Commons Attribution License, which permits unrestricted use, distribution, and reproduction in any medium, provided the original work is properly cited.

\begin{abstract}
Spin-orbit Rashba effect applies a torque on the magnetization of a ferromagnetic nanostrip in the case of structural inversion asymmetry, also affecting the steady domain wall motion induced by a spin-polarized current. This influence is here analytically studied in the framework of the extended Landau-Lifshitz-Gilbert equation, including the Rashba effect as an additive term of the effective field. Results of previous micromagnetic simulations and experiments have shown that this field yields an increased value of the Walker breakdown current together with an enlargement of the domain wall width. In order to analytically describe these results, the standard travelling wave ansatz for the steady domain wall motion is here adopted. Results of our investigations reveal the impossibility to reproduce, at the same time, the previous features and suggest the need of a more sophisticated model whose development requires, in turn, additional information to be extracted from ad hoc micromagnetic simulations.
\end{abstract}

\section{Introduction}

Magnetization dynamics in nanodevices has been intensely investigated in the last decades as it provides a wide variety of technological applications in the area of storage and logic devices. Formerly, the manipulation of the magnetic configuration was typically achieved by means of external magnetic fields but the contemporary demand of miniaturizing storage devices and of increasing their capacity would have required higher and higher fields. An alternative method, realized by using spin-polarized currents, was outlined by the discovery of spin-transfer torque effect $[1,2]$. Theoretical and experimental studies, therefore, examined magnetization dynamics due to the simultaneous action of external magnetic fields and electric currents [3-5] as well as current-driven dynamics at zero field $[6,7]$.

Among the different geometries used for spintronic devices, a more recent attention is directed to magnetic nanowires and strips [8-22]. Such thin ferromagnetic structures turn out to be relevant for the realization of oscillators and high-density memories with low energy consumption [9]. In particular, their behavior and applications are strictly connected to the motion of magnetic domain walls (DWs), namely, the continuous transition regions that separate two uniformly and oppositely magnetized domains $[9,10]$. Also, in this case, DW dynamics can be activated by means of an external field or an electric current. Independently of the nature of the source term, it has been widely demonstrated that, by varying the strength of such a driving source, the DW motion experiences two different dynamical regimes. At low fields (or currents), the equilibrium wall structure is rigidly shifted along the nanostrip (or nanowire) axis, leading to a "steady" regime of high DW mobility. Above a critical field (or current) value, named Walker breakdown, a regime of lower DW mobility takes place and the internal deformation is so strong that the wall structure is altered giving rise to a 
periodical alternation of Bloch and Néel DW structures. Such a dynamics is generally referred to as "precessional" regime [11].

DW motion can be also strongly modified by spin-orbit interaction [12] that takes place in the case of structural inversion asymmetry of the nanodevice. Some previous works [13-18], in particular, pointed out that this effect, induced by the flow of an electric current through the material, acts as an effective field. This contribution, which was named Rashba field, produces two main consequences: (1) a (Bloch-like) DW structure is stabilized as the steady regime turns broadened up to higher current densities so allowing higher DW velocities; (2) the DW width enlarges with the increase of the current-induced Rashba field.

From the theoretical viewpoint, DW motion in ferromagnetic thin layers is ruled by the extended LandauLifshitz-Gilbert (ELLG) equation including the currentdriven spin-torque effects [20-24]. Travelling wave solutions for the ELLG equation have been recently obtained, providing a strong theoretical support for both experimental and numerical results $[20,22]$. It has been also demonstrated that the inclusion of a different dissipation function into the ELLG equation, usually referred to as "dry-friction," gives a good description of DW dynamics in the presence of crystallographic defects and structural disorder $[20,22]$.

In this work, we propose to analytically study, by using a one-dimensional mathematical model, the steady DW motion in ferromagnetic nanostrips subject to the action of spin-polarized currents and Rashba fields. In particular, we investigate on the appropriateness of using a standard travelling wave ansatz describing a Bloch DW structure which rigidly shifts under the action of the external source. In order to validate the developed model, these analytical results are then compared, at qualitative level, with those arising from recent numerical and experimental investigations. The model also accounts for the nonlinear dry-friction dissipation function in order to evaluate how such a contribution affects the current-driven steady DW motion in the presence of a Rashba field.

\section{The Analytical One-Dimensional Model: Results and Discussion}

As depicted in Figure 1, a ferromagnetic nanostrip can be represented by a rectangular prism of length $l$, width $w$, and thickness $t$ along $\mathbf{c}_{x}, \mathbf{c}_{y}, \mathbf{c}_{z}$ axes, respectively, with $l \gg$ $w>t$. Let us assume that an electric current density $\mathbf{J}=$ $J c_{x}$, constant in time and uniform in space, is applied to the device along the $\mathbf{c}_{x}$ axis. Under the hypothesis that the Rashba field does not modify the equilibrium configuration obtained in its absence, we assume that a $180^{\circ} \mathrm{DW}$ of width $\delta$ is nucleated at the center of the structure with the magnetization vector that rotates between the state $(0,0,1)$ at $x \rightarrow-\infty$ and the opposite one $(0,0,-1)$ at $x \rightarrow \infty$. These uniformly magnetized states, far away from the wall location, are hence supposed to be directed towards the easy axis $\mathbf{c}_{z}=\mathbf{e}$, namely, the energetically preferred direction of spontaneous magnetization.

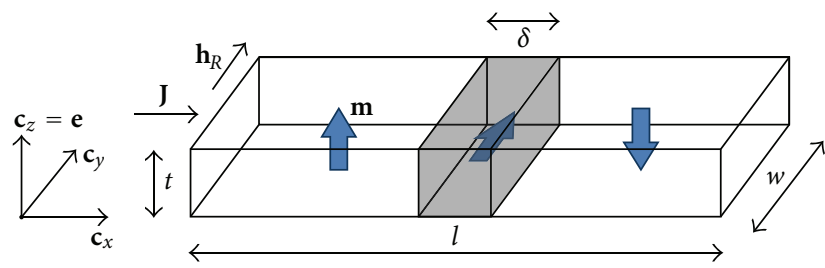

FIGURE 1: Schematics of a ferromagnetic nanostrip exhibiting a Bloch DW.

Current-driven DW dynamics in such a thin layer is described by the ELLG equation [20-24]:

$$
\dot{\mathbf{m}}=\gamma\left(\mathbf{h}_{\text {eff }} \wedge \mathbf{m}\right)+\mathbf{t}_{d}+\mathbf{t}_{\mathrm{st}},
$$

where the over-dot denotes time derivation, $\mathbf{m}=\mathbf{M} / M_{S}$ is the unit vector along the local magnetization and all the field vectors are normalized with respect to the saturation magnetization $M_{S}$. The constant $\gamma=M_{S} \mu_{0} \gamma_{e}$ is expressed in terms of the vacuum magnetic permeability $\mu_{0}$ and the gyromagnetic ratio $\gamma_{e}=g e / m_{e}$, being $g$ the Landé factor, $e$ the electron charge, and $m_{e}$ the electron mass. The first term in the right-hand side of (1) describes the precession of magnetization $\mathbf{m}$ around the direction of the effective

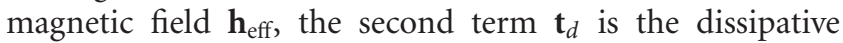
torque representing energy dissipation, and $\mathbf{t}_{\mathrm{st}}$ corresponds to the current-induced spin torque.

The effective magnetic field $\mathbf{h}_{\text {eff }}=-\partial W / \partial \mathbf{m}$, calculated as the variational derivative of the free energy density $W$, accounts for external $\mathbf{h}_{\text {ext }}$, exchange $\mathbf{h}_{\text {exc }}$, demagnetizing $\mathbf{h}_{\mathrm{dmg}}$, anisotropy $\mathbf{h}_{\mathrm{an}}$, and Rashba $\mathbf{h}_{R}$ fields:

$$
\mathbf{h}_{\mathrm{eff}}=\mathbf{h}_{\mathrm{ext}}+\mathbf{h}_{\mathrm{exc}}+\mathbf{h}_{\mathrm{dmg}}+\mathbf{h}_{\mathrm{an}}+\mathbf{h}_{R},
$$

As our attention focuses on the influence of the currentinduced Rashba field, we will limit our analysis to the zerofield configuration:

$$
\mathbf{h}_{\text {ext }}=0 \text {. }
$$

The exchange field can be written as

$$
\mathbf{h}_{\mathrm{exc}}=A \frac{\partial^{2} \mathbf{m}}{\partial x^{2}}
$$

being $A$ related to the exchange constant $A_{\mathrm{ex}}$ of the material through

$$
A=\frac{2 A_{\mathrm{ex}}}{\mu_{0} M_{S}^{2}} .
$$

The demagnetizing field can be approximated by considering the only diagonal terms of the corresponding tensor which relates the field to the magnetization [11]:

$$
\mathbf{h}_{\mathrm{dmg}}=-N_{x}\left(\mathbf{m} \cdot \mathbf{c}_{x}\right) \mathbf{c}_{x}-N_{y}\left(\mathbf{m} \cdot \mathbf{c}_{y}\right) \mathbf{c}_{y}-N_{z}(\mathbf{m} \cdot \mathbf{e}) \mathbf{e}
$$

where $N_{x}, N_{y}$, and $N_{z}$ are the demagnetizing factors satisfying the normalization condition $N_{x}+N_{y}+N_{z}=1$. These coefficients depend both on the shape (assumed to be 
a rectangular prism) and on the dimensions $(w \times t \times \delta)$ of the DW.

We also assume that the strip is made by a material exhibiting a high perpendicular magnetic anisotropy, so that we can express

$$
\mathbf{h}_{\mathrm{an}}=\beta(\mathbf{m} \cdot \mathbf{e}) \mathbf{e},
$$

where $\beta \gg 1$ is proportional to the anisotropy constant $K$ of the material through

$$
\beta=\frac{2 K}{\mu_{0} M_{S}^{2}} .
$$

Finally, the Rashba field is given by [13-18]:

$$
\mathbf{h}_{R}=\frac{\alpha_{R} P}{\mu_{0} \mu_{B} M_{S}^{2}}(\mathbf{e} \wedge \mathbf{J})
$$

being $\alpha_{R}$ the Rashba parameter, $P$ the polarization factor of the current, and $\mu_{B}$ the Bohr magneton. The expression (9) can be also written in compact form as

$$
\mathbf{h}_{R}=\chi u \mathbf{c}_{y}
$$

where $\chi$ accounts for the Rashba effect, $\chi=\left(2 e / g \mu_{0} \mu_{B}^{2} M_{S}\right) \alpha_{R}$, and the spin-torque velocity $u$ accounts for the applied current, $u=\left(g \mu_{B} P / 2 e M_{S}\right) J$.

The dissipative torque $\mathbf{t}_{d}$ is here described by the phenomenological Gilbert-like expression that also includes a dry-friction damping function [19-22]:

$$
\mathbf{t}_{d}=\mathbf{m} \wedge\left[\alpha_{G}+\frac{\zeta}{|\dot{\mathbf{m}}|}\right] \dot{\mathbf{m}}
$$

Being $\alpha_{G}$ the classical linear damping coefficient and $\zeta$ the phenomenological dry-friction parameter (for more details on the dry-friction formulation, together with its practical justification within the equation of motion, see [20-22]).

The spin transfer torque $\mathbf{t}_{\mathrm{st}}$ is given by

$$
\mathbf{t}_{\mathrm{st}}=u(-1+\eta \mathbf{m} \wedge) \frac{\partial \mathbf{m}}{\partial x}
$$

where $\eta$ is the phenomenological nonadiabatic spin-torque parameter [10].

Taking into account (3)-(12), (1) becomes

$$
\begin{aligned}
\dot{\mathbf{m}} & -\left[\alpha_{G}+\frac{\zeta}{|\dot{\mathbf{m}}|}\right](\mathbf{m} \wedge \dot{\mathbf{m}}) \\
& =\left(\gamma \mathbf{h}_{\mathrm{eff}}-u \mathbf{m} \wedge \frac{\partial \mathbf{m}}{\partial x}-\eta u \frac{\partial \mathbf{m}}{\partial x}\right) \wedge \mathbf{m}
\end{aligned}
$$

with

$$
\begin{aligned}
\mathbf{h}_{\mathrm{eff}}= & A \frac{\partial^{2} \mathbf{m}}{\partial x^{2}}+\left(\beta-N_{z}\right)(\mathbf{m} \cdot \mathbf{e}) \mathbf{e} \\
& -N_{x}\left(\mathbf{m} \cdot \mathbf{c}_{x}\right) \mathbf{c}_{x}-N_{y}\left(\mathbf{m} \cdot \mathbf{c}_{y}\right) \mathbf{c}_{y}+\chi u \mathbf{c}_{y} .
\end{aligned}
$$

Let us now make a transformation from the Cartesian to the spherical frame, so that it is possible to express the local magnetization as:

$$
\mathbf{m}=\cos \varphi \sin \vartheta \mathbf{c}_{x}+\sin \varphi \sin \vartheta \mathbf{c}_{y}+\cos \vartheta \mathbf{e} .
$$

From (13)-(15), we therefore obtain a system of two secondorder partial differential equations:

$$
\begin{aligned}
\dot{\vartheta}+ & {\left[\alpha_{G}+\left(\dot{\vartheta}^{2}+\sin ^{2} \vartheta \dot{\varphi}^{2}\right)^{-1 / 2} \zeta\right] \sin \vartheta \dot{\varphi} } \\
= & A \gamma \sin \vartheta \frac{\partial^{2} \varphi}{\partial x^{2}}+2 A \gamma \cos \vartheta \frac{\partial \varphi}{\partial x} \frac{\partial \vartheta}{\partial x} \\
& +\gamma\left(N_{x}-N_{y}\right) \sin \varphi \cos \varphi \sin \vartheta \\
& -\eta u \sin \vartheta \frac{\partial \varphi}{\partial x}-u \frac{\partial \vartheta}{\partial x}+\gamma \chi u \cos \varphi \\
\sin \vartheta \dot{\varphi} & -\left\{\left[\alpha_{G}+\left(\dot{\vartheta}^{2}+\sin ^{2} \vartheta \dot{\varphi}^{2}\right)-1 / 2 \zeta\right] \dot{\vartheta}\right\} \\
= & -A \gamma \frac{\partial^{2} \vartheta}{\partial x^{2}}+A \gamma \sin \vartheta \cos \vartheta\left(\frac{\partial \varphi}{\partial x}\right)^{2} \\
& +\gamma \sin \vartheta \cos \vartheta\left(\beta-N_{z}+N_{x} \cos ^{2} \varphi+N_{y} \sin ^{2} \varphi\right) \\
& -u \sin \vartheta \frac{\partial \varphi}{\partial x}+\eta u \frac{\partial \vartheta}{\partial x}-\gamma \chi u \sin \varphi \cos \vartheta .
\end{aligned}
$$

Since it was demonstrated that the previous system admits analytical solutions in the form of travelling waves [19-22], we search for such solutions apt to describe the DW motion within the steady regime. In particular, in order to reduce the system (16) to a couple of ordinary differential equations, we study the appropriateness of adopting the commonly used travelling wave ansatz $\vartheta=\vartheta(x-v t)$, where the DW velocity $v$ is assumed to be a positive constant, and $\varphi=\varphi_{0}=$ constant. By using this strategy, we get

$$
\begin{aligned}
& (u-v) \vartheta^{\prime}=\gamma \cos \varphi_{0}\left[\left(N_{x}-N_{y}\right) \sin \varphi_{0} \sin \vartheta+\chi u\right] \\
& \left(\alpha_{G} v-\eta u\right) \vartheta^{\prime}+\hat{\zeta}=-\gamma A \vartheta^{\prime \prime} \\
& +\gamma \sin \vartheta \cos \vartheta\left(\beta-N_{z}+N_{x} \cos ^{2} \varphi_{0}+N_{y} \sin ^{2} \varphi_{0}\right) \\
& -\gamma \chi u \sin \varphi_{0} \cos \vartheta
\end{aligned}
$$

where $\hat{\zeta}=\zeta \operatorname{sign}\left(v \vartheta^{\prime}\right)$, while the prime denotes the derivative with respect to the travelling wave variable $x-v t$ and the boundary conditions take the Dirichlet form $\vartheta(-\infty)=0$, $\vartheta(+\infty)=\pi$. We can recast $(17)_{1}$ in the following form:

$$
\vartheta^{\prime}=\Gamma_{0}(\sin \vartheta+\rho),
$$

where

$$
\begin{gathered}
\rho=\frac{\gamma \chi u \cos \varphi_{0}}{(u-v) \Gamma_{0}} \\
\Gamma_{0}=\frac{\gamma\left(N_{x}-N_{y}\right) \cos \varphi_{0} \sin \varphi_{0}}{u-v} .
\end{gathered}
$$


In $(19)_{1}$ the parameter $\rho$ accounts for the presence of the Rashba field. For what concerns $(19)_{2}$, let us remind that, in the absence of the Rashba field and nonlinear dissipations, we recover the classical definition of DW width $\Gamma_{0}^{-1}=\delta$ $[10,11,20]$ with

$$
\delta=\sqrt{\frac{A}{\beta-N_{z}+N_{y}+\left(N_{x}-N_{y}\right) \cos ^{2} \varphi_{0}}} .
$$

Let us also notice that the knowledge of the DW width requires, as shown in (20), the value of the demagnetizing factors $\left(N_{x}, N_{y}, N_{z}\right)$. On the other hand, as discussed after (6), the demagnetizing factors can be computed once the DW width is known. This apparent conflict is generally solved by determining the DW width by means of alternative methods (e.g., by extracting it either from the profile of the travelling wave computed numerically and/or analytically, or from experiments).

Substituting (18) in $(17)_{2}$, after some algebraic steps, leads to

$$
\widehat{M} \sin \vartheta+\widehat{Q} \sin \vartheta \cos \vartheta+\widehat{S} \cos \vartheta+\widehat{P}=0
$$

where

$$
\begin{gathered}
\widehat{M}=\Gamma_{0}\left(\alpha_{G} v-\eta u\right) \\
\widehat{Q}=\gamma\left[A \Gamma_{0}^{2}-\left(\beta-N_{z}+N_{x} \cos ^{2} \varphi_{0}+N_{y} \sin ^{2} \varphi_{0}\right)\right] \\
\widehat{S}=\gamma \chi u\left(\sin \varphi_{0}+\frac{\gamma A \Gamma_{0} \cos \varphi_{0}}{u-v}\right) \\
\hat{P}=\left(\alpha_{G} v-\eta u\right) \frac{\gamma \chi u \cos \varphi_{0}}{u-v}+\hat{\zeta},
\end{gathered}
$$

By performing the average of (21) over the DW width (i.e., for $0 \leq \vartheta \leq \pi$ ) and taking into account that the terms defined in (22) do not depend on $\vartheta$, it is possible to derive the following expression for the DW velocity $v$ as a function of the current-dependent spin-torque velocity $u$ :

$$
v=\frac{\left(\eta \chi \Gamma_{0} /\left(N_{x}-N_{y}\right)\right) u^{2}+\left(2 \eta \Gamma_{0} / \pi\right) u-\hat{\zeta}}{\left(\alpha_{G} \chi \Gamma_{0} /\left(N_{x}-N_{y}\right)\right) u+\left(2 \alpha_{G} \Gamma_{0} / \pi\right)},
$$

where it is supposed to deal with a DW of Bloch type $\left(\varphi_{0}=\right.$ $\pi / 2)[19,20]$.

As pointed out in some previous works [20-22], the inclusion of a dry-friction dissipation generally yields the steady DW motion to take place for values of the input stimulus which overcome a well-defined threshold. Equation (23) gives also the possibility to determine such a threshold current, defined as the minimum current value which satisfies the condition $v \geq 0$. In order to properly investigate on this aspect (and to emphasize the sole effect of the Rashba field), let us consider the two separate cases corresponding to the presence and the absence of the dry-friction dissipation function.

2.1. DW Dynamics in the Absence of Dry-Friction $(\zeta=0)$. If we exclude the additive dry friction term in the dissipation function, (23) simply reduces to

$$
v=\frac{\eta}{\alpha_{G}} u
$$

that, interestingly, matches exactly the current-driven steady DW velocity derived in the absence of the Rashba field [20, 22]. It is also straightforward to notice that, in the perfect adiabatic case $(\eta=0)$, no DW motion occurs. Equation (24) also implies that the threshold current is null:

$$
u_{\text {th }}=0 \text {, }
$$

so that the DW motion takes place for any nonnull value of the applied current. Results coming from (24) and (25), which clearly claim that the Rashba field does not modify the DW velocity (and, in turn, the DW mobility, defined as the ratio between the velocity and the input current) and the threshold current, satisfactorily agree with the recent experimental $[14,15]$ and numerical $[17,18]$ investigations. However, since these studies pointed out an increase of the Walker breakdown value and an enlargement of the DW width, we perform further investigations in this direction to validate the appropriateness of our initial conjectures.

In order to determine the Walker breakdown, from the definition $(19)_{1}$, we can write

$$
\sin 2 \varphi_{0}=\frac{2 \Gamma_{0}(u-v)}{\gamma\left(N_{x}-N_{y}\right)}
$$

that implies

$$
u-\frac{\gamma}{2 \Gamma_{0}}\left|N_{x}-N_{y}\right| \leq v \leq u+\frac{\gamma}{2 \Gamma_{0}}\left|N_{x}-N_{y}\right| .
$$

Let us remember that the left and the right implications of (27) are representative of the so-called lower and upper Walker breakdown conditions, respectively [20-22]. They define the range of the input source in which the steady DW motion takes place.

By comparing the expression of the DW velocity (24) and the breakdown condition (27) with those derived in the absence of the Rashba field [20,22], we report no differences which, at first, would lead to conclude that the inclusion of this field contribution has no influence on the DW dynamics at all. Nonetheless, as mentioned previously, the works carried out in $[15,17]$ have shown that, in a framework with Rashba field and no internal disorder, in spite of the DW mobility that is unchanged, the upper Walker breakdown is increased (the lower breakdown brings a nonnegligible contribution only in the presence of nonlinear dissipations [22]). Since our results do not allow to apparently satisfy this latter property, we therefore ask on the reasons of this discrepancy. We believe that the answer has to be searched in the numerical values of the parameters appearing in the expression of the Walker breakdown. In detail, we can hypothesize that the Rashba field has changed the modulus of the quantity $\gamma\left|N_{x}-N_{y}\right| / 2 \Gamma_{0}$, making it somehow larger. In particular, considering that the expression of $\Gamma_{0}(19)$ is not formally affected, the only way to obtain such an increase is that the demagnetizing factors, strictly related to the DW width, are varied. In order to find out if our conjecture is correct, we search for the expression of the DW profile by 
integrating (18). The resolving procedure strictly depends on the parameter $\rho$ that can be recast in the form:

$$
\rho=\frac{\chi u}{\left(N_{x}-N_{y}\right)} .
$$

For completeness, let us remember first that, in the case $\rho=$ 0 , corresponding to the absence of Rashba field, the solution is in the classical form $[19,20]$ :

$$
\vartheta=2 \arctan \left[e^{\Gamma_{0}(x-v t)}\right]
$$

where $\Gamma_{0}$ indeed equals the inverse of the DW width.

Let us discuss, now, the solutions obtained as a function of the strength of the quantity $|\rho|$.

For $|\rho|<1$, the solution can be expressed as

$$
\vartheta=\arccos \frac{1-f^{2}(x-v t)}{1+f^{2}(x-v t)},
$$

where the expression of the function $f$ can be found in (99)(102) of [19] and in (35) of [20]. In this case, the DW profile (30) is distorted with respect to the classical case, as shown in Figure 4 of [19], and, in particular, the DW width increases with increasing $\rho$. Under this circumstance, therefore, the analytical model confirms the enlargement of the DW width which was highlighted in a previous work [18]. Nevertheless, this increase of the DW width changes, in turn, the values of the demagnetizing factors, in such a way that $N_{x}$ approaches $N_{y}$ leading to an overall decrease of the quantity $\left|N_{x}-N_{y}\right|$, so lowering the Walker breakdown value. This latter evidence is thus in contradiction with the other expected feature of the dynamics under investigation.

For this reason, we can hypothesize that the correct solution has to be searched for $|\rho|>1$. The solution in this case is

$$
\vartheta=2 \arctan \left\{\frac{\sqrt{\rho^{2}-1} \tan \left[\Gamma_{0} \sqrt{\rho^{2}-1}(x-v t)+\kappa\right]-1}{\rho}\right\}
$$

being $\kappa$ the integration constant

$$
\kappa=\frac{1}{\sqrt{\rho^{2}-1}} \arctan \left(\frac{\rho+1}{\sqrt{\rho^{2}-1}}\right)
$$

that has to be chosen in such a way the variable $\vartheta$, evaluated at the center of the DW, is null. It should be indeed mentioned that, in this case, the travelling wave solution does not allow to satisfy the Dirichlet boundary conditions, so that the solution is only locally valid, namely, in the proximity of the center of the DW.

In order to estimate the orders of magnitude of the quantities involved in the model and to validate our assumption, we carry out a numerical evaluation of the travelling wave profile. For this reason, we take into account the parameter setup proposed in [17]. In detail, we consider a magnetic nanostrip of thickness $t=3 \mathrm{~nm}$ and $w=120 \mathrm{~nm}$,

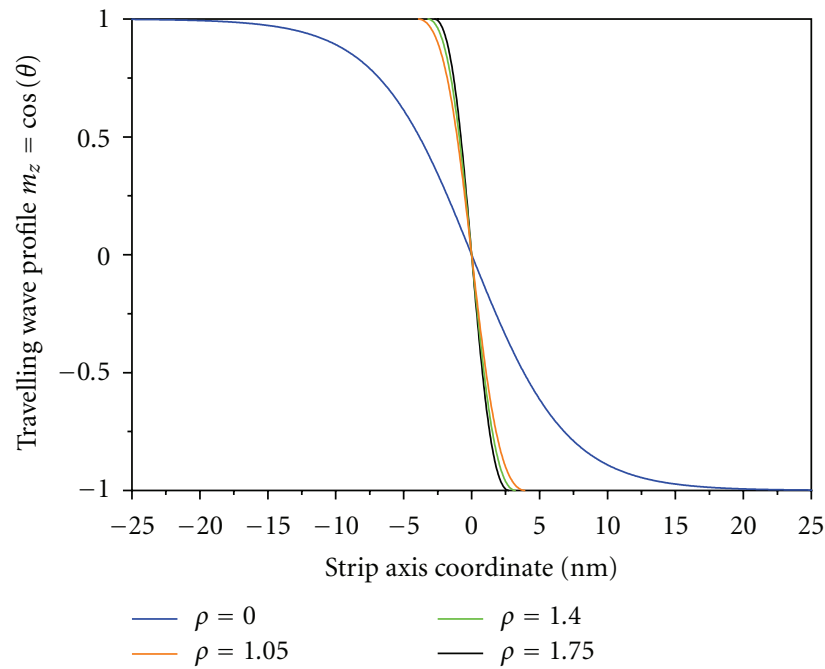

(a)

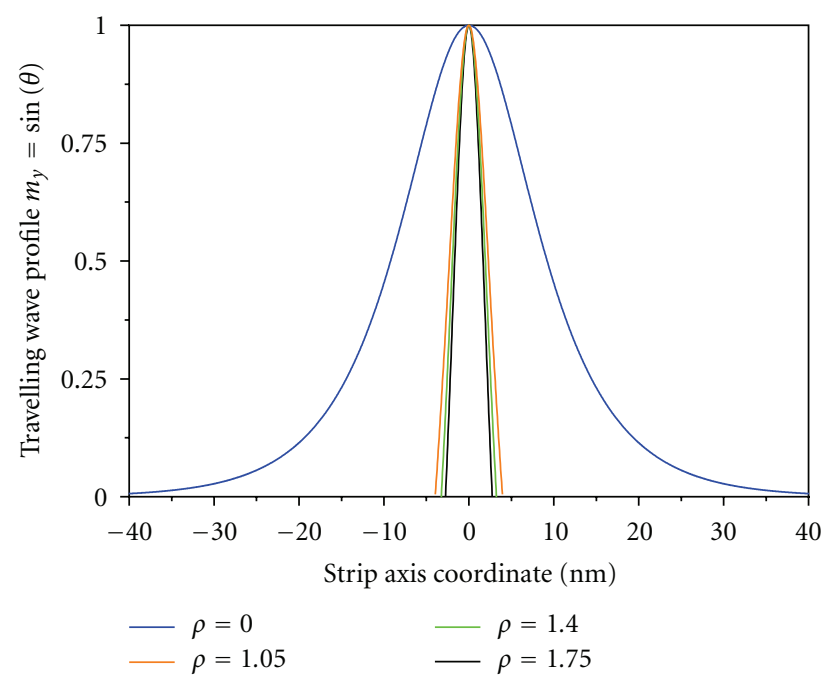

(b)

FIGURE 2: Comparison among the travelling wave profiles with and without Rashba field $(|\rho|>1$ and $\rho=0$, resp.) computed analytically by using (31)-(32).

saturation magnetization $M_{S}=3 \times 10^{5} \mathrm{~A} / \mathrm{m}$, Landé factor $g=2$, exchange constant $A=10^{-11} \mathrm{~J} / \mathrm{m}$, anisotropy constant $K=2 \times 10^{5} \mathrm{~J} / \mathrm{m}^{3}$, Gilbert damping constant $\alpha_{G}=0.2$, polarization factor $P=0.5$, nonadiabatic parameter $\eta=0.4$.

Figure 2 shows the profile of the Bloch DW (characterized by $\left.\varphi_{0}=\pi / 2\right)$, as its components $m_{z}=\cos \vartheta$ and $m_{y}=\sin \vartheta\left(m_{x}\right.$ is null everywhere), for $\rho=0$ (namely, with no Rashba field) and $|\rho|>1$ (with Rashba field), as deduced from (31)-(32). It is clear that, in this case, the presence of the Rashba field would strongly modify the DW profile and width, making this latter about ten times narrower. It can be also appreciated that the DW width is only slightly affected by variations of $\rho$, higher than unity.

Starting from these results, we evaluate the new demagnetizing factors corresponding to the modified situation. 


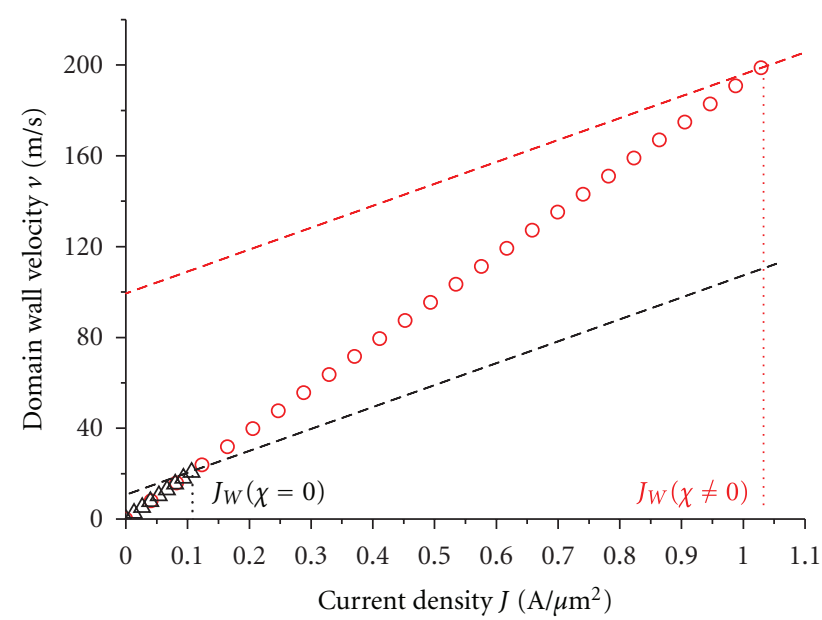

$\triangle$ DW velocity for $\chi=0 \quad---$ Upper breakdown for $\chi=0$

○ DW velocity for $\chi \neq 0 \quad--$ Upper breakdown for $\chi \neq 0$

FIgURE 3: Current-driven DW velocity in the steady dynamic regime with and without Rashba field ( $\chi \neq 0$ and $\chi=0$, resp.) computed analytically by using (24) together with the upper breakdown condition (27).

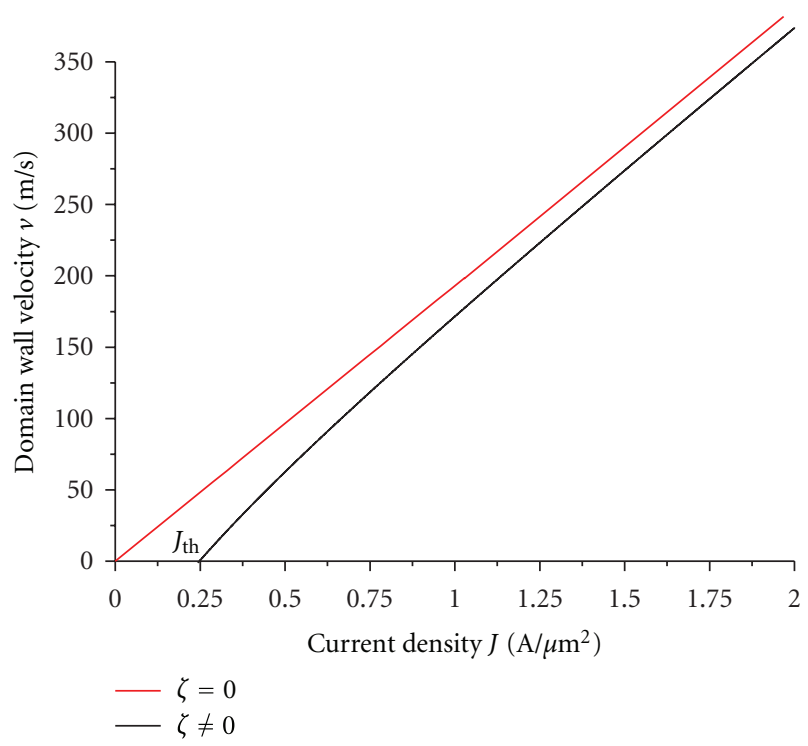

FIgURE 4: Current-driven DW velocities in the steady dynamic regime with and without the inclusion of the dry-friction dissipation function in the mathematical model $(\zeta \neq 0$ and $\zeta=0$, resp.). The limit of Walker has not been considered in this figure in order to better emphasize the nonlinear behavior of the DW velocity influenced by internal disorder, $\zeta \neq 0$, with respect to the case $\zeta=0$. Walker breakdown conditions, indeed, are not influenced by $\zeta$.

Because of the DW width is reduced, the difference $\left|N_{x}-N_{y}\right|$ increases, leading to an increase of the Walker breakdown. For instance, such a difference is equal to 0.046 for $\rho=0$ and 0.445 for $|\rho|>1$. The resulting increase of the Walker breakdown value and the enlargement of the range of steady DW motion are depicted in Figure 3 (obtained by using (24) together with (27)). When no Rashba field is considered, namely, $\chi=0$ (i.e., $\rho=0$ ), the Walker breakdown current is $J_{W}=0.11 \mathrm{~A} / \mu \mathrm{m}^{2}$. Under the influence of the Rashba effect, instead, the upper Walker limit increases, making the steady regime possible up to a higher current $J_{W}(\chi \neq 0)=$ $1.04 \mathrm{~A} / \mu \mathrm{m}^{2}$. From a direct inspection of Figure 3, it should be also noticed that, in both cases, there is no threshold current and the DW mobility is not affected by the Rashba field.

Summarizing, the case $|\rho|>1$ leads to the expected raise of the Walker breakdown value but negates the enlargement of the DW width.

We can state, therefore, that the classical travelling wave ansatz for the ELLG equation $(\vartheta=\vartheta(x-v t)$ with $v=$ constant and $\varphi=$ constant) cannot be satisfactorily used to reproduce the overall effects of the Rashba field on the steady DW motion in ferromagnetic nanostrip.

2.2. DW Dynamics in the Presence of Dry Friction $(\zeta \neq 0)$. The usage of a dry-friction dissipation function already turned to be useful to model the effects of crystallographic defects, structural disorder, including surface roughness, on the DW motion $[20,21]$. In particular, it was demonstrated that the inclusion of such a friction mechanism leads to the appearance of a threshold below which no DW motion can take place, whereas the DW mobility is not affected in the above-threshold regime. In the present work, we would like to test these two properties when the dry-friction dissipation acts simultaneously to the Rashba field. To this aim, (23) is plotted in Figure 4. As it is expected, also in this case, the dry friction causes the motion to occur for current values larger than a given threshold current $J_{\text {th }}$ (in the figure, $J_{\text {th }}=0.25 \mathrm{~A} / \mu \mathrm{m}^{2}$ for $\zeta=2 \times 10^{-2} \gamma$ ). On the other hand, the DW velocity, which followed a linear trend in the absence of Rashba field (independently of the presence of dry friction), now exhibits a nonlinear dependence on the input current which approximate, for large current values, the velocity obtained in the case $\zeta=0$. It is interesting to notice that the same result was qualitatively obtained in experiments [16] as well as in numerical simulations [17] that accounted for thermal effects and roughness. However, the corresponding travelling wave solutions would suffer from the same incompatibility with respect to numerical and experimental observations.

\section{Conclusions}

In this work, we have analyzed the bias-field-free currentdriven DW motion in a ferromagnetic nanostrip subject to the Rashba field and dry-friction dissipation. The study has been mathematically carried out by modifying the extended Landau-Lifshitz-Gilbert equation with the inclusion of the Rashba contribution into the effective field. The standard travelling wave ansatz generally used for the equation of motion, within the steady regime, does not succeed in confirming simultaneously both the key features revealed in recent numerical and experimental observations : increase of the Walker breakdown value and enlargement of the DW width. 
This result suggests that the system (16) has to be solved by using a different approach. We believe that, due to the transversal component of the effective field induced by the Rashba field, the hypothesis $\varphi=$ constant, together with the Dirichlet boundary conditions $\vartheta(-\infty)=0$ and $\vartheta(\infty)=$ $\pi$, does not apply in this case. For this reason, one could consider that the angle $\varphi$ exhibits an analogous travelling wave dependence $\varphi=\varphi(x-v t)$ and that, due to the symmetry of the problem, Neumann boundary conditions $\vartheta^{\prime}( \pm \infty)=0$ and $\varphi^{\prime}( \pm \infty)=0$ should be satisfied instead. By imposing such constraints, we get the following nonlinear system of coupled ordinary differential equations:

$$
\begin{aligned}
&(u-v) \vartheta^{\prime}-\left(\vartheta^{\prime 2}+\sin ^{2} \vartheta \varphi^{\prime 2}\right)^{-1 / 2} \zeta \nu^{2} \sin \vartheta \varphi^{\prime} \\
&- 2 A \gamma \cos \vartheta \vartheta^{\prime} \varphi^{\prime}-A \gamma \sin \vartheta \varphi^{\prime \prime} \\
&+\left(\eta u \sin \vartheta-\alpha_{G} v \sin \vartheta\right) \varphi^{\prime} \\
&-\gamma\left(N_{x}-N_{y}\right) \sin \varphi \cos \varphi \sin \vartheta \\
&-\gamma \chi u \cos \varphi=0 \\
& \gamma \vartheta^{\prime \prime}+\left(\alpha_{G} v-\eta u\right) \vartheta^{\prime}+\left(\vartheta^{\prime 2}+\sin ^{2} \vartheta \varphi^{\prime 2}\right)^{-1 / 2} \zeta v^{2} \vartheta^{\prime} \\
&-A \gamma \sin \vartheta \cos \vartheta \varphi^{\prime 2}+(u-v) \sin \vartheta \varphi^{\prime} \\
&-\gamma \sin \vartheta \cos \vartheta\left(\beta-N_{z}+N_{x} \cos ^{2} \varphi+N_{y} \sin ^{2} \varphi\right) \\
&+\gamma \chi u \sin \varphi \cos \vartheta=0 .
\end{aligned}
$$

However, solving this system without simplifying assumptions is not trivial at all. For example, the missing information could be argued from ad hoc micromagnetic simulations which should provide the accurate profile of the travelling wave variable and their dependence on the strength of the Rashba field. Therefore, we strongly encourage numerical investigations in this direction to overcome this issue.

\section{Acknowledgment}

G. Consolo gratefully acknowledges support from GNFMINdAM.

\section{References}

[1] J. C. Slonczewski, "Current-driven excitation of magnetic multilayers," Journal of Magnetism and Magnetic Materials, vol. 159, no. 1-2, pp. L1-L7, 1996.

[2] L. Berger, "Emission of spin waves by a magnetic multilayer traversed by a current," Physical Review B, vol. 54, no. 13, pp. 9353-9358, 1996.

[3] A. N. Slavin and P. Kabos, "Approximate theory of microwave generation in a current-driven magnetic nanocontact magnetized in an arbitrary direction," IEEE Transactions on Magnetics, vol. 41, no. 4, pp. 1264-1273, 2005.
[4] G. Finocchio, O. Ozatay, L. Torres, M. Carpentieri, G. Consolo, and B. Azzerboni, "Micromagnetic modeling of magnetization reversal in nano-scale point contact devices," IEEE Transactions on Magnetics, vol. 43, no. 6, pp. 2938-2940, 2007.

[5] G. Consolo and V. Puliafito, "Analytical and micromagnetic study of nonlinear amplitude modulation in spintronic modulators," IEEE Transactions on Magnetics, vol. 46, no. 6, Article ID 5467373, pp. 2063-2066, 2010.

[6] X. Zhu and J. G. Zhu, "Bias-field-free microwave oscillator driven by perpendicularly polarized spin current," IEEE Transactions on Magnetics, vol. 42, pp. 2670-2672, 2006.

[7] V. Puliafito, B. Azzerboni, G. Consolo, G. Finocchio, L. Torres, and L. L. Diaz, "Micromagnetic modeling of nanocontact spin-torque oscillators with perpendicular anisotropy at zero bias field," IEEE Transactions on Magnetics, vol. 44, no. 11, pp. 2512-2515, 2008.

[8] N. Vernier, D. A. Allwood, D. Atkinson, M. D. Cooke, and R. P. Cowburn, "Domain wall propagation in magnetic nanowires by spin-polarized current injection," Europhysics Letters, vol. 65, no. 4, pp. 526-532, 2004.

[9] D. A. Allwood, G. Xiong, C. C. Faulkner, D. Atkinson, D. Petit, and R. P. Cowburn, "Magnetic domain-wall logic," Science, vol. 309, no. 5741, pp. 1688-1692, 2005.

[10] A. Thiaville, Y. Nakatani, J. Miltat, and Y. Suzuki, "Micromagnetic understanding of current-driven domain wall motion in patterned nanowires," Europhysics Letters, vol. 69, no. 6, pp. 990-996, 2005.

[11] A. Mougin, M. Cormier, J. P. Adam, P. J. Metaxas, and J. Ferré, "Domain wall mobility, stability and Walker breakdown in magnetic nanowires," Europhysics Letters, vol. 78, no. 5, Article ID 57007, 2007.

[12] A. Manchon and S. Zhang, "Theory of spin torque due to spinorbit coupling," Physical Review B - Condensed Matter and Materials Physics, vol. 79, no. 9, Article ID 094422, 2009.

[13] K. Obata and G. Tatara, "Current-induced domain wall motion in Rashba spin-orbit system," Physical Review B, vol. 77, no. 21, Article ID 214429, 2008.

[14] I. M. Miron, G. Gaudin, S. Auffret et al., "Current-driven spin torque induced by the Rashba effect in a ferromagnetic metal layer," Nature Materials, vol. 9, no. 3, pp. 230-234, 2010.

[15] I. M. Miron, T. Moore, H. Szambolics et al., "Fast currentinduced domain-wall motion controlled by the Rashba effect," Nature Materials, vol. 10, no. 6, pp. 419-423, 2011.

[16] J. Ryu, S. B. Choe, and H. W. Lee, "Magnetic domain-wall motion in a nanowire: depinning and creep," Physical Review $B$, vol. 84, Article ID 075469, 2011.

[17] E. Martinez, "The influence of the Rashba field on the current-induced domain wall dynamics: a full micromagnetic analysis, including surface roughness and thermal effects," Journal of Applied Physics, vol. 111, Article ID 07D302, 2012.

[18] E. Martinez, "Micromagnetic analysis of the Rashba field on current-induced domain wall propagation," Journal of Applied Physics, vol. 111, Article ID 033901, 2012.

[19] P. Podio-Guidugli and G. Tomassetti, "On the steady motions of a flat domain wall in a ferromagnet," European Physical Journal B, vol. 26, no. 2, pp. 191-198, 2002.

[20] G. Consolo, C. Currò, E. Martinez, and G. Valenti, "Mathematical modeling and numerical simulation of domain wall motion in magnetic nanostrips with crystallographic defects," Applied Mathematical Modelling, vol. 36, no. 10, pp. 48764886, 2012. 
[21] G. Consolo and E. Martinez, "The effect of dry friction on domain wall dynamics: a micromagnetic study," Journal of Applied Physics, vol. 111, Article ID 07D312, 3 pages, 2012.

[22] G. Consolo and G. Valenti, "Travelling wave solutions of the one-dimensional extended Landau-Lifshitz-Gilbert equation with nonlinear dry and viscous dissipations," Acta Applicandae Mathematicae, 2012.

[23] L. D. Landau and E. Lifshitz, "On the theory of the dispersion of magnetic permeability in ferromagnetic bodies," Physikalische Zeitschrift der Sowietunion, vol. 8, pp. 153-169, 1935.

[24] W. F. Brown, Micromagnetics, Krieger, Huntington, NY, USA, 1963. 

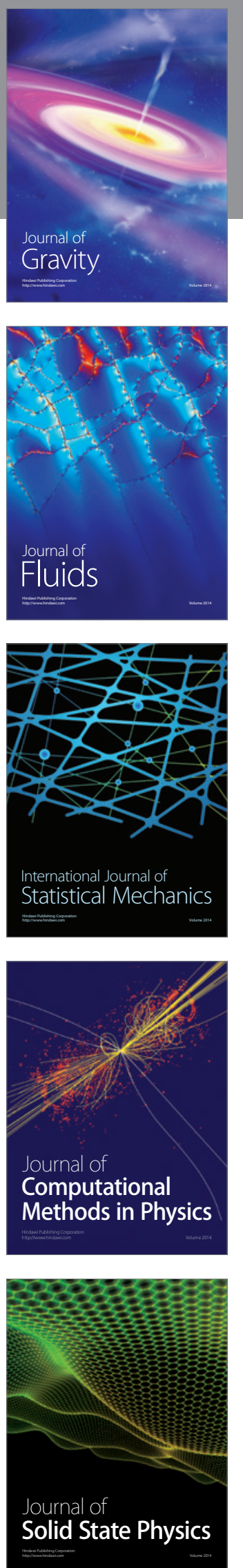

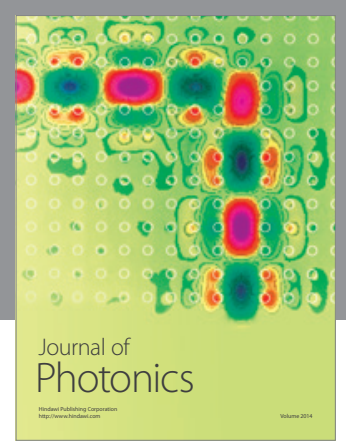

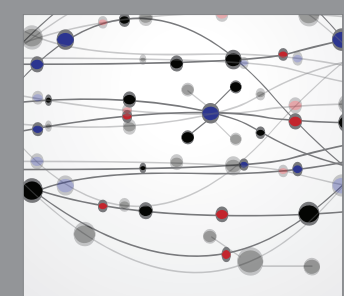

The Scientific World Journal
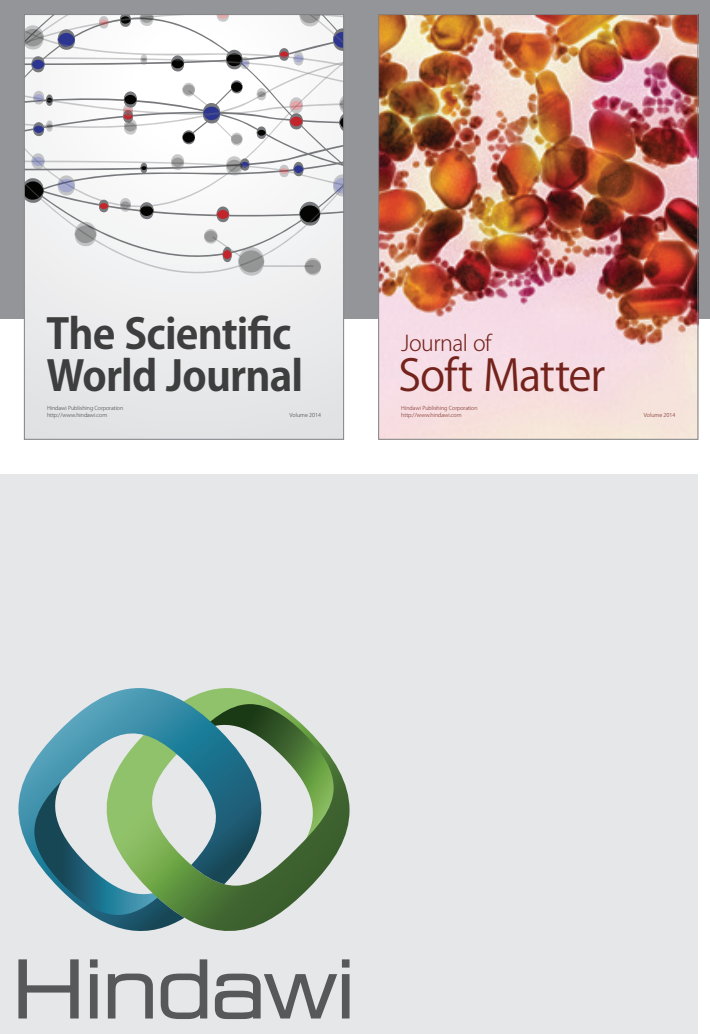

Submit your manuscripts at

http://www.hindawi.com
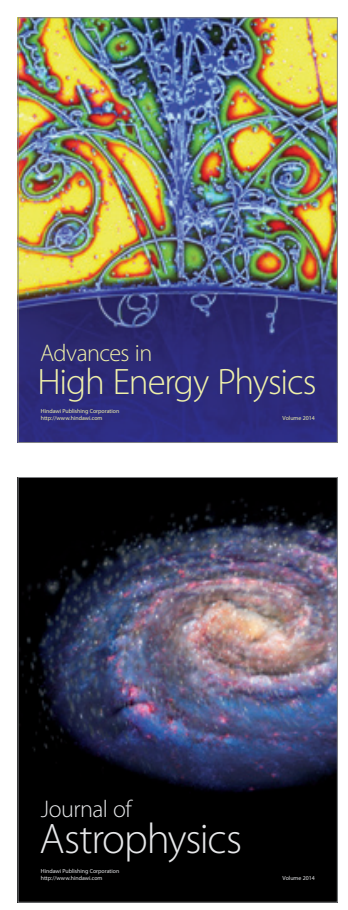
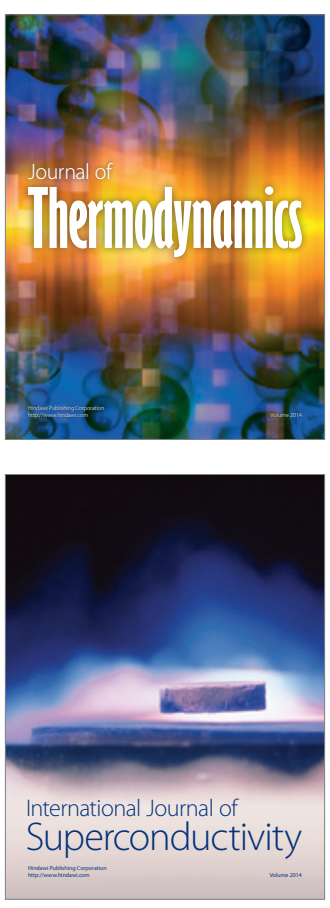
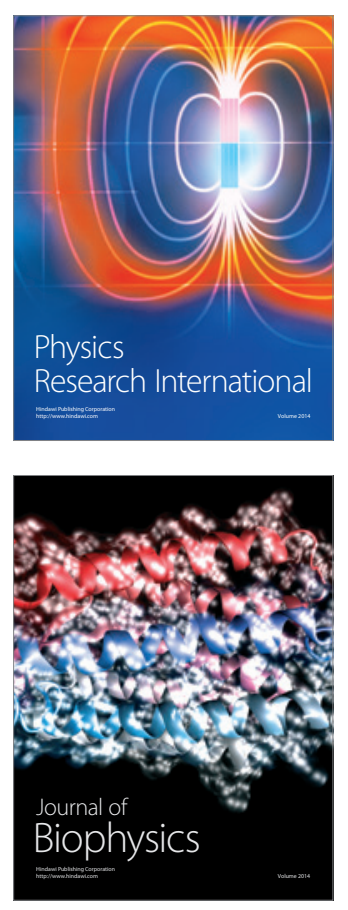
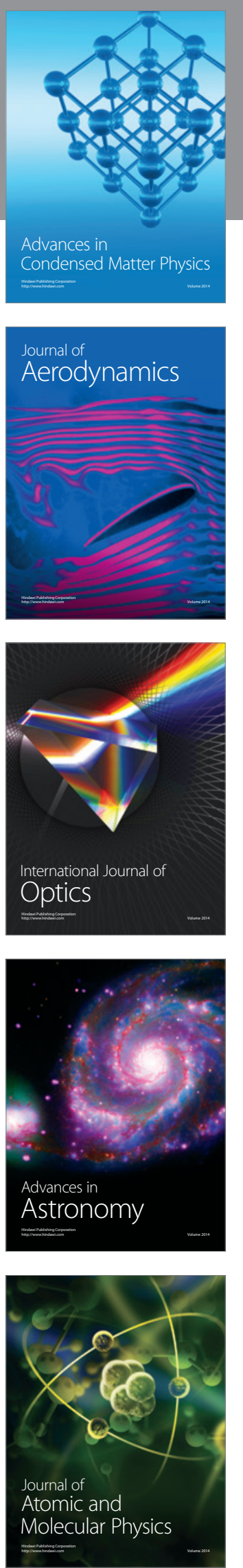